\title{
EFECTO ANTIMICROBIANO DEL EXTRACTO ETANÓLICO DE CAESALPINIA ESPINOSA (TARA) AL $60 \%$, SOBRE EL ENTEROCOCCUS FAECALIS.
}

\section{ANTIMICROBIAL EFFECT OF A 60\% ETHANOLIC EXTRACT OF CAESALPINIA ESPINOSA(TARA) ON ENTEROCOCCUS FAECALIS}

\author{
Bornaz-Arenas Vanessa L . ${ }^{*}$ Bornaz-Acosta Juan G. ${ }^{2}$ \\ ${ }^{1}$ Docente de la Univ. Andina Néstor Cáceres Velasquez. \\ ${ }^{2}$ Docente Univ. Nacional Jorge Basadre Ghroman. \\ *bornazzz@hotmail.com
}

\begin{abstract}
Resumen
El Enterococcus Faecalis se considera una de las cepas más resistente al grupo de soluciones Desinfectantes habitualmente manipuladas en los tratamientos de ConductosOBJETIVO: Establecer la producción de efecto inhibitorio de la Caesalpinia espinosa (Tara) sobre la cepa Enterococcus Faecalis MATERIALES Y MÉTODOS: Se realizó una siembra de la cepa ATCC 29212 (Enterococcus Faecalis) en placas con Agar Cerebro Corazón, luego se colocó en la superficie sensidiscos de $5 \mathrm{~mm}$ de diámetro con $\mathrm{NaClO}$ al 5,25\% como control y Caesalpinia espinosa (Tara) al $60 \%$ como grupo experimental, para cada sustancia se utilizó 24 muestras; luego se incubó las placas en cámara de anaerobiosis a $37^{\circ} \mathrm{C}$, posteriormente se tomó medidas a las 24; 48 y 72 horas del halo inhibitorio resultante.RESULTADOS: Los datos obtenidos fueron ordenados y procesados, indicando el promedio de la medida del halo inhibitorio tanto de la Caesalpinia espinosa resultando este mayor que el formado por el Hipoclorito de Sodio, se aplicó un Test de Normalidad para los datos obtenidos con ambas sustancias, determinando que dichos datos tenían una distribución normal. Aplicando posteriormente $\mathrm{T}$ de Student $(\mathrm{p}<0,05)$, dando como resultado diferencia estadísticamente significativa entre los datos obtenidos para ambas sustancias.CONCLUSIÓN: La Caesalpinia espinosa, demostró tener efecto antibacteriano frente al Enterococcus Faecalis, sus medidas fueron variadas y en la mayoría superiores a Hipoclorito de Sodio.
\end{abstract}

Palabras clave: Antimicrobiano, Fracaso Endodóntico, Enterococcus faecalis, Caesalpinia espinosa, Hipoclorito de Sodio .

\begin{abstract}
AMI:Enterococcus Faecalis is considered one of the strains more resistant to the solutions group disinfectants commonly manipulated in the treatments of ducts The objective of this research was to establish the production of inhibitory effect of Caesalpinia spinosa (tare) on the Enterococcus Faecalis strain MATERIALS AND METHODS: Was a seeding of the strain ATCC 29212 (Enterococcus Faecalis) plates with Agar brain heart, then it is placed on the surface of 5 mm diameter discs with $\mathrm{NaClO}$ to the 5,25\% as control and Caesalpinia spinosa (tare) to 60\% as a group experimental, for each substance was used 24 samples; then incubated in anaerobic Chamber plates at $37^{\circ} \mathrm{C}$, was subsequently taken measures 24; 48 and 72 hours after the resulting inhibitory halo RESULTS: Data were sorted and processed, indicating the average of the measurement of both the Caesalpinia spinosa inhibitory halo resulting this greater than that formed by sodium hypochlorite, applied a Test of normality for data obtained with both substances, determining that such data had a normal distribution. Then applying Student's $T(p<0,05)$, giving as result statistically significant difference between the data obtained for both substances CONCLUSION:The Caesalpinia spinosa, proved to have antibacterial effect against Enterococcus Faecalis, its measures were varied and mostly higher than sodium hypochlorite.
\end{abstract}

Key words: Antimicrobial, endodontic failure, Enterococcus faecalis, Caesalpinia spinosa, sodium hypochlorite. 


\section{INTRODUCCIÓN}

En el mundo más del $90 \%$ de la población sufre de caries sin embargo el paciente no le presta la atención debida en sus estadios incipientes por lo que sigue progresando hasta la afectación de los tejidos pulpares, siendo el posible tratamiento para poder conservar la pieza en boca realizar un tratamiento de conducto, cuyo procedimiento es para la remoción del tejido pulpar y así eliminar el daño. ${ }^{2,8}$ Actualmente existen sustancias medicamentosas utilizados en esta terapia, con el fin de disminuir o eliminar infecciones bacterianas y algunos de ellos fracasan. ${ }^{3,4}$ En la actualidad, según estudios preliminares, una de las bacterias más tenaces al uso de sustancias antimicrobianas utilizadas en endodoncia es por ello que es responsable de la mayoría fracasos endodónticos es el Enterococcus faecalis. ${ }^{5-7}$

Actualmente los tratamientos odontológicos buscan reducir los índices de caries, índices de inadecuada higiene oral aplicando métodos, estrategias y programas preventivos de caries. Sin embargo estos aplicativos darán resultados en el futuro mientras que los procedimientos clínicos resuelven el daño que ya está presente: por lo tanto es importante considerar aplicar tratamientos conservadores, eliminando los microorganismos más agresivos presentes como en el caso del tratamiento Endodóntico y lograr así un éxito cercano al $100 \%{ }^{8,9}$ Actualmente, la medicina natural está en apogeo por los múltiples beneficios que nos dan las plantas en es especial de nuestro país, además de tener menos efectos secundarios que las sustancias sintéticas producidas en laboratorio. La Caesalpina espinosa, más conocida como tara, es una planta de la cual se obtiene una sustancia, que no solo será capaz de disminuir o eliminar microorganismos, ${ }^{10-12}$ sino que también es un poderoso cicatrizante según estudios preliminares, siendo un producto natural tiene menor cantidad de efectos adversos. ${ }^{13}$ Es significativo, que se realicen investigaciones para determinar el efecto antimicrobiano de sustancias de origen natural tomándolas como alternativa a las que ya existen en el mercado, siendo las sustancias naturales las más indicadas, debido que presentan menor cantidad de efectos adversos. Estos antecedentes nos ha motivado a estudiar el efecto antimicrobiano de Caesalpina espinosa.

\section{MATERIALES Y MÉTODOS}

Tipo de Investigación: De laboratorio, longitudinal Nivel de Investigación: Comparativo Experimental Diseño: Factorial de Grupos

Identificación de los grupos Los Grupos serán distribuidos en las placa petri, 24 muestras para cada uno de los grupos; Grupo experimental: Caesalpinia Espinosa al $60 \%$, grupo control: Hipoclorito de Sodio al 5,25\% Criterios Incluyentes Cepa Enterococcus Faecalis Criterios Excluyentes Cepa contaminada de Enterococcus Faecalis Descripción de la Técnica Se utilizó como materiales: un extracto de Caesalpina espinosa (Tara) al $60 \%$ para el desarrollo del grupo experimental; la cepa ATCC 29212 (Enterococcus faecalis) y una solución de $\mathrm{HClO}$ al 5,25\% para el grupo control. Se sembró la cepa en placas con Agar Cerebro Corazón, luego se colocó sensidiscos de $5 \mathrm{~mm}$ de diámetro con Hipoclorito de Sodio al 5,25\% como control y Caesalpinia espinosa (Tara) al $60 \%$ como grupo experimental; se utilizó 24 muestras por cada una de las sustancias a investigar; se colocó las placas en incubación en cámara de anaerobiosis a $37^{\circ} \mathrm{C}$, tomándose medidas de halo inhibitorio tomando medidas a las 24; $48 \mathrm{y}$ 72 horas con una regla tipo vernier.

Para la sustancia HIPOCLORITO DE SODIO el promedio fue de 2,63896 en la primera medición; por lo tanto generó un halo inhibitorio de $2,64 \mathrm{~mm}$ en la siembra microbiológica. En la medición de 24 horas 2,73958 y en la tercera lectura 2,82625; lo que indica que el valor promedio va aumentando ligeramente de una medición a otra. Se concluye que el diámetro del día 1 posee una leve asimetría, luego el día 2 se va tornando menos asimétrico y el día 3 se va aproximando más a una distribución normal. La Desviación Estándar en la primera fue de 0,670042 en la segunda de 0,653748 , y en la tercera de 0,703713 , con respecto al mínimo los datos se distribuyen en un rango de 1,250 a 1,500 para el primer día y el tercer día respectivamente, lo que índica que los valores mínimos del día 1 al día 3 fueron aumentando progresivamente, para el máximo los valores fueron de 3,750 para el día 1 para el día 2 la medida fue de 4,025 y 4,075 para el día 3 de lo cual se puede deducir que el valor máximo fue aumentando del día 1 al 3 lo que indica que fue una progresión constante. Los valores para la mediana fueron de 2,51500 para el día 1, 2,52500 para el día 2 y para el día 3 de 3,02500. En la asimetría se obtuvo para el día 1 un valor de 0,070 para el día 2 una valor de 0,401 y para el día 3 un valor de 0,301 . Los valores en la curtosis para la primera medición fueron - 0,649 ; para la segunda medición fueron $-0,989$ y para la tercera medición fue $-0,890$.

\section{RESULTADOS}

Para la sustancia Caesalpinia espinosa nos da diámetros de la primera lectura de 6,01875; lo que interpreta que el halo inhibitorio fue 6. Posteriormente el halo fue de 6,18854; y finalmente en la tercera lectura fue de 6,33021; por lo que se determina que el halo va aumentando gradualmente.Se concluye que la medida del día 1 posee una leve asimetría, el día 2 se va volviendo menos asimétrico y el día 3 se va aproximando más a una distribución normal. La desviación Estándar en la primera medición fue de 0,720479; en la segunda de 0,674778; y en la tercera fue de 0,686238; con respecto al mínimo los datos se distribuyen en un rango de 4,500 a 5,025; para el primer día y el tercer día respectivamente, lo cual determina que los valores del día 1 al día 3 fueron aumentando progresivamente, para el máximo los valores fueron de 7,525 para el día 1; 7,575 para el día 2 y 7,525 para el día 3 . De lo cual se deduce los valores aumentaron y luego se mantuvieron igual al día 1 . Los valores 


\begin{tabular}{lllllllll}
\hline & N & Promedio & $\begin{array}{l}\text { Desviación } \\
\text { Estándar }\end{array}$ & Mínimo & Máximo & Mediana & Asimetría & Curtosis \\
\hline Día 1 & 24 & 6,01875 & 0,720479 & 4,500 & 7,525 & 6,0500 & $-0,021$ & 0,150 \\
Día 2 & 24 & 6,18854 & 0,74778 & 5,000 & 7,575 & 6,25000 & $-0,097$ & $-0,180$ \\
Día 3 & 24 & 6,33021 & 0,686238 & 5,025 & 7,525 & 6,3500 & $-0,038$ & $-0,705$ \\
\hline
\end{tabular}

Tabla 1: ESTADÍSTICOS DESCRIPTIVOS DEL GRUPO EXPERIMENTAL

\begin{tabular}{lllllllll}
\hline & N & Promedio & $\begin{array}{l}\text { Desviación } \\
\text { Estándar }\end{array}$ & Mínimo & Máximo & Mediana & Asimetría & Curtosis \\
\hline Día 1 & 24 & 2,63896 & 0,670042 & 1,250 & 3,750 & 2,51500 & 0,070 & $-0,649$ \\
Día 2 & 24 & 2,73958 & 0,653748 & 1,750 & 4,025 & 2,52500 & 0,401 & $-0,989$ \\
Día 3 & 24 & 2,82625 & 0,703713 & 1,500 & 4,075 & 3,02500 & 0,031 & $-0,890$ \\
\hline
\end{tabular}

Tabla 2: ESTADÍSTICOS DESCRIPTIVOS DEL GRUPO CONTROL

para la mediana fueron de 6,05000 para el día 1; 6,25000 para el día 2 y para el día 3 de 6,35000. En la asimetría se obtuvo para el día 1 un valor de -0,021; para el día 2 un valor de $-0,097$ y para el día 3 un valor de -0,038. Los valores en la curtosis para la primera medición fueron 0,150; para la segunda medición fueron $-0,180$ y para la tercera medición fue $-0,705$.

Tabla 3.- Comparación primera lectura

\begin{tabular}{ccc}
\hline Día 1 & $\begin{array}{c}\text { Grupo } \\
\text { Experimental }\end{array}$ & Control \\
\hline Promedio & 6,019 & 2,639 \\
Mediana & 6,050 & 2,515 \\
Moda & 6,250 & 2,000 \\
Mínimo & 4,500 & 1,250 \\
Máximo & 7,525 & 3,750 \\
Desv, Est, & 0,720 & 0,670 \\
\hline \multicolumn{3}{c}{ T de Student $\mathrm{p}<0,05$}
\end{tabular}

Tabla 4.- Comparación segunda lectura

\begin{tabular}{ccc}
\hline Día 2 & $\begin{array}{c}\text { Grupo } \\
\text { Experimental }\end{array}$ & Control \\
\hline Promedio & 6,189 & 2,740 \\
Mediana & 6,250 & 2,525 \\
Moda & 6,250 & 2,525 \\
Mínimo & 5,000 & 1,750 \\
Máximo & 7,575 & 4,025 \\
Desv, Est, & 0,675 & 0,654 \\
\hline \multicolumn{3}{c}{ T de Student $\mathrm{p}<0,05$}
\end{tabular}

Los valores del promedio para el grupo Caesalpinia Espinosa es 6,019 y para el grupo Hipoclorito es de 2,639; determina que la discrepancia entre la media de ambos halos inhibitorios fue de por lo menos $3,38 \mathrm{~mm}$, siendo el promedio de halos inhibitorios para la sustancia experimental mayor que la media de los halos inhibitorios de la sustancia control, la mediana para el control es 2,515 y para el experimental es 6,050 ; con respecto a la moda para el grupo del Hipoclorito es de 2,000 y para la Caesalpinia Espinosa es 6,250; el valor mínimo para el grupo control es de 1,250 y para el grupo experimental es de 4,500; el valor máximo para el grupo control es de 3,750 y para el grupo experimental es de 7,525; tanto para los valores máximos y mínimos para el primer día de toma de datos, el grupo experimental Caesalpinia espinosa presentaba valores bastante altos a comparación del grupo control y con respecto a la desviación estándar los valores fueron de 0,670; para el hipoclorito y 0,720; para la Caesalpinia espinosa. Los promedios son muy disímiles y ambas distribuciones estadísticas tienen calores variados para los datos obtenidos a las 24 horas(Fig 1).

Tabla 5.- Comparación tercera lectura

\begin{tabular}{ccc}
\hline Día 3 & $\begin{array}{c}\text { Grupo } \\
\text { Experimental }\end{array}$ & Control \\
\hline Promedio & 6,330 & 2,826 \\
Mediana & 6,350 & 3,025 \\
Moda & 6,000 & 2,075 \\
Mínimo & 5.025 & 1,500 \\
Máximo & 7,525 & 4,075 \\
Desv, Est, & 0,686 & 0,704 \\
\hline \multicolumn{3}{c}{ T de Student $\mathrm{p}<0,05$}
\end{tabular}

El Promedio para el grupo de Experimental es 6,189 y para el grupo Control es 2,740; lo cual determina que la diferencia entre las medias de los halos inhibitorios fue de $3.449 \mathrm{~mm}$, el grupo experimental obtuvo un mayor promedio, la mediana para el Grupo control es 2,525 y para el grupo experimental es 6,250; con respecto a la moda para el grupo del Hipoclorito es de 2,525 y para la Caesalpinia espinosa es de 6,250; el valor mínimo para el grupo control es de 1,750 y para el grupo experimental es de 5,000; el valor máximo para el grupo control es de 4,025 y para el grupo experimental es de 7,575; tanto para los valores máximos y mínimos para el segundo día de toma de datos, el grupo experimental Caesalpinia espinosa presentaba valores bastante altos a comparación del grupo control con respecto a la desviación estándar los valores fueron de 0,654; para el hipoclorito y 
0,675; para la Caesalpinia espinosa.En la segunda lectura los promedios y rangos de datos, demuestran tener diferencia estadísticamente significativa, ambas distribuciones estadísticas tiene rangos muy variados(Fig 2).

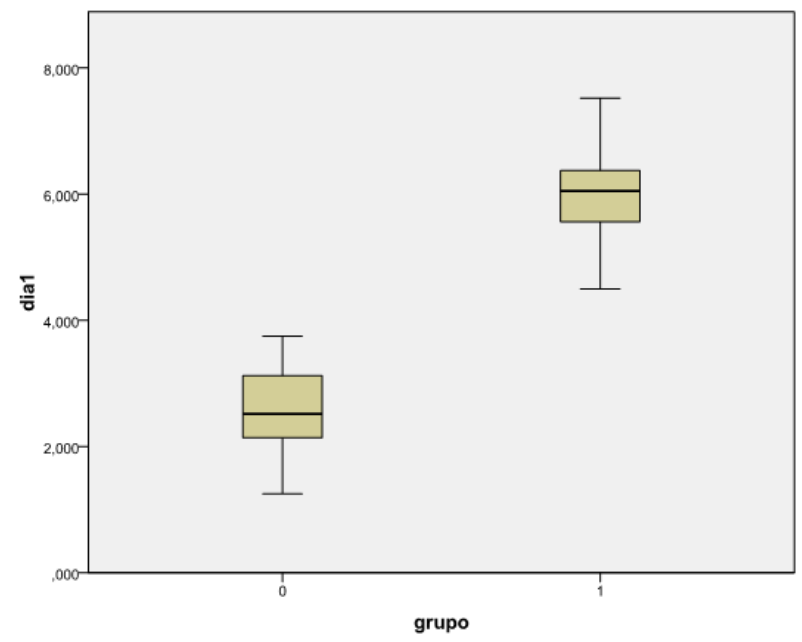

Fig. 1. COMPARACIÓN ENTRE EL GRUPO EXPERIMENTAL CAESALIPINIA ESPINOSA Y GRUPO CONTROL HIPOCLORITO DE SODIO EN LA PRIMERA LECTURA.

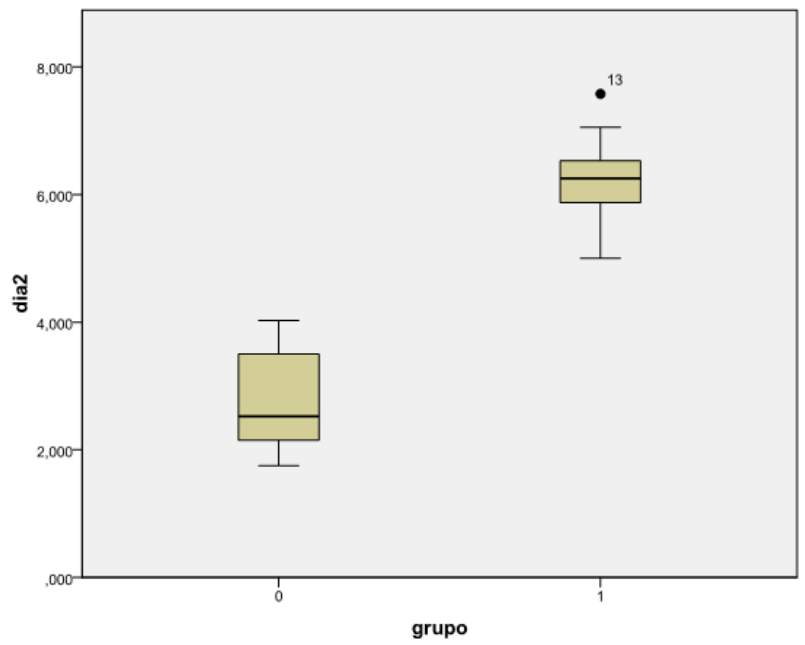

Fig. 2. COMPARACIÓN ENTRE EL GRUPO CONTROL Y GRUPO EXPERIMENTAL EN LA SEGUNDA LECTURA.

Los valores del promedio para el grupo Caesalpinia espinosa es 6,330 y para el grupo Hipoclorito es 2,826; por lo que se con concluye que la discrepancia entre los promedios de ambos halos inhibitorios fue de 3,504 mm, los halos inhibitorios para grupo experimental resultaron ser mayores que el promedio de los halos inhibitorios del grupo control, la mediana para el Grupo control es 3,025 y para el grupo experimental es de 6,350; con respecto a la moda para

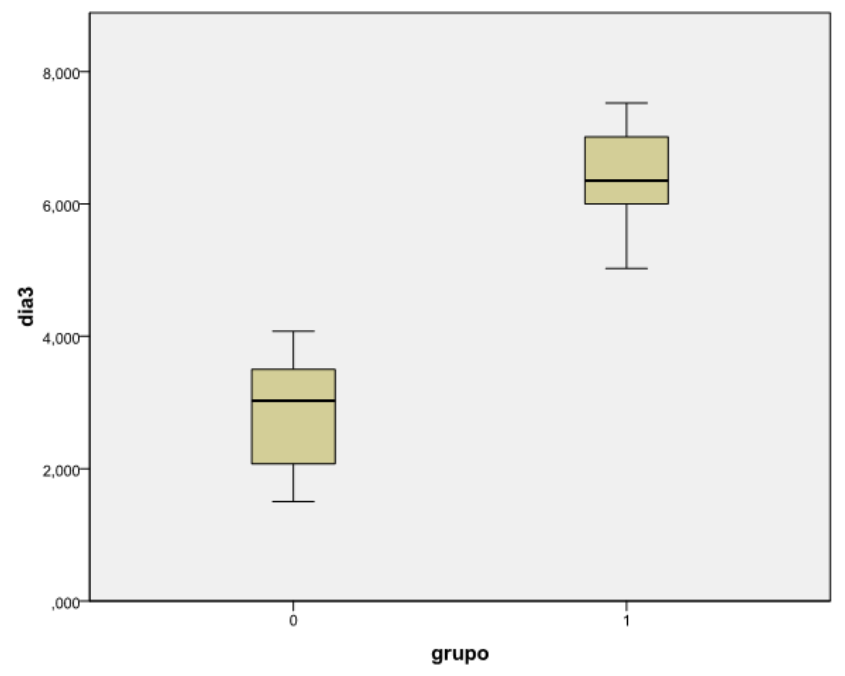

Fig. 3. COMPARACIÓN ENTRE EL GRUPO EXPERIMENTAL Y GRUPO EN LA TERCERA LECTURA.

el grupo del Hipoclorito es de 2,075 y para la Caesalpinia Espinosa es de 6,000; el valor mínimo para el grupo control es de 1,500 y para el grupo experimental es de 5,025; el valor máximo para el grupo control es de 4,075 y para el grupo experimental es de 7,525; tanto para los valores máximos y mínimos para el primer día de toma de datos, el grupo experimental Caesalpinia espinosa presentaba valores bastante altos a comparación del grupo control y con respecto a la desviación estándar los valores fueron de 0,704; para el hipoclorito y 0,686; para la Caesalpinia espinosa.Se observa que los promedio son muy diferentes y ambas distribuciones estadísticas tienen rangos múltiples para los valores obtenidos el día 3; demostrando tener diferencia estadísticamente significativa(Fig 3).

\section{DISCUSIÓN}

Sánchez Ruiz y colaboradores. ${ }^{13}$ En su investigación sobre actividad microbiana de Hipoclorito de sodio al $5 \%$ en la dilución 1:1 (2,5\%) dio como resultado que la actividad antimicrobiana no duró hasta las 72 horas. Podría deberse a que se trabajó con hipoclorito diluido, por lo tanto el alcance antimicrobiano se redujo, comparándolo con nuestro estudio el efecto antimicrobiano si duro 72 horas por que se trabajó con hipoclorito al $5 \%$. Espinel Pinzon Mercy. ${ }^{14} \mathrm{Su}$ estudio fue realizado en 30 premolares que fueron infectados con enterococcus Faecalis, a los cuales se les colocó 4 combinaciones de $\mathrm{NaOCl}$ y EDTA. Los resultados obtenidos fueron que ninguna de las sustancias utilizadas ya sea solas o mezcladas con el quelante lograron eliminar la cepa en cuestión. Uno de los factores que podría haber dado este resultado es que la investigación se realizó en diente natural ya sus compleja estructura anatómica pudo haber afectado el alcance del hipoclorito, a comparación de los resultados 
obtenidos en la presente investigación donde si se aprecia un efecto antimicrobiano en la cepa escogida. Kloucek, P y col. ${ }^{15} \mathrm{Su}$ estudio fue realizado con extractos etanólicos al $80 \%$ de nueve plantas obtenidos por maceración durante 5 días, una de ellas fue la Caesalpinia. spinosa utilizando sus vainas; además se utilizó cinco cepas Gram positivas y tres Gram negativas. Los resultados obtenidos fue que para el Enterococcus Faecalis se observó una CIM de 0,5 $\mathrm{mg} / \mathrm{ml}$, para Bacillus cereus fue de 8 y de 16; para las demás bacterias, lo cual determina la especificidad y gran poder antimicrobiano de esta sustancia natural, lo fue corroborado por nuestro estudio.

\section{CONCLUSIONES}

- La Caesalpinia Espinosa al $60 \%$ logró un halo inhibitorio de $6,33 \mathrm{~mm}$; en promedio con una desviación típica de 0,68 frente al Enterococcus Faecalis • El HClO al 5,25\% logró un halo inhibitorio de $2,82 \mathrm{~mm}$ en promedio con una desviación típica de 0,7; frente al Enterococcus Faecalis • Se encontró diferencia estadísticamente significativa entre el efecto antimicrobiano de la Caesalpinia Espinosa (Tara) al $60 \%$ y el $\mathrm{HClO}$ al $5,25 \%$ en el halo inhibitorio del Enterococcus Faecalis, esto con un margen de error del $5 \%$. • La hipótesis nula fue rechazada, debido a que el Halo inhibitorio de la Tara al $60 \%$ obtuvo mayores valores que el hipoclorito de Sodio al $5,25 \%$ por lo que su efecto antimicrobiano fue mayor para nuestra sustancia experimental en el halo inhibitorio del Enterococcus Faecalis

\section{Referencias}

1 Gonzalez MA, Gonzalez N. Infecciones bacterianas de origen pulpar y periodontal. Oral Med Oral Pathol Oral Surg. 2004

2 Portenier I, Waltimo T. Haapasalo M. Enterococcus Fecalis and endash; the root canal survivor and "star" in post -treatment disease. Endod. Topics 2003;6 : 135-139

3 Stuart C. Schwartz S. Beeson T, Owatz C. Enterococcus Faecalis: Its role in root canal treatment failure and current concepts in retreatment. J Endod. 2006;32 : 93-98

4 Attin et al, El hipoclorito de sodio disuelve tejido necrótico, no tejido vital. J International Endod. 2002

5 George S, Kishen A, Song K. The Role of Environmental Changes on Monospecies Biofilm Formation on Root Canal Wall by Enterococcus faecalis. J Endod. 2005; 31: 867-72.

6 Lima K, Fava L, Siqueira J. Susceptibilities of Enterococcus faecalis biofilms to some antimicrobial medications. J Endod. 2001; 27: 616-9.

7 Mc Hugh C, Zhang P, Michalek S, Eleazer P. Ph required to kill Enterococcus faecalis in vitro. J Endod. 2004;30 : 218219

8 Frais, S. Gulabivala K. Some factors affecting the concentration of avaible chlorine in commercial sources of sodium hypochlorite. Int Endod J. 2001
9 García, G., Olivo, R, Ochoa, C. Complicaciones con el hipoclorito de sodio (NaOCL) al entrar en contacto con los tejidos periradiculares. Univers Odont. 2001. 21:26-29.

0 Estrela C, Estrela CRA, Barbin EL, Spanó JL, Marchesan MA, Pécora JD. Mechanism of action of Sodium Hypochlorite. Branz Dent J 2002;13: 113-117

1 Carson KR, Goodell GG, McClanahan SB. Comparison of the Antimicrobial Activity of Six Irrigants on Primary Endodontics Patogens. J Endodon 2005; 3: 471-473

12 Barriga, C. Cultivo y Aprovechamiento de la Tara, Caesalpinia espinosa, en la Región Andina. Informe Técnico. Lima 2008

13 Sánchez Ruiz J, Olivo, R y Garcia M. The effect of passive ultrasonic activation of $2 \%$ chlorhexidine or $5.25 \%$ sodium hypochlorite irrigant on residual antimicrobial activity in root canals. J of Endod Sept 2003; 29: 78-81.

14 Espinel Pinzon M. Efecto antimicrobiano de hipoclorito de sodio y EDTA sobre Enterococcus Faecalis. Tesis para optar el grado de magister en Salud Pública UNSA, 2011.

15 Kloucek P, Lima K y Fava L. Evaluation of the antibacterial activities of calcium hydroxide, chlorhexidine, medicament. A Clinical and Laboratory Study. J Endodont, May, 2007.

Recibido: 13 de enero de 2018.

Aceptado: 17 de marzo de 2018. 
